\title{
Editorial
}

\section{Collaboration and Optimization for Multimedia Communications}

\author{
Jianwei Huang, ${ }^{1} \mathrm{Zhu} \mathrm{Li}^{2}$ and Qian Zhang ${ }^{3}$ \\ ${ }^{1}$ Department of Information Engineering, The Chinese University of Hong Kong, New Territory, Hong Kong SAR, China \\ ${ }^{2}$ Department of Computing, The Hong Kong Polytechnic University, Kowloon, Hong Kong SAR, China \\ ${ }^{3}$ Department of Computer Science and Engineering, Hong Kong University of Science and Technology, Kowloon, \\ Hong Kong SAR, China \\ Correspondence should be addressed to Jianwei Huang, jwhuang@ie.cuhk.edu.hk
}

Received 27 February 2008; Accepted 27 February 2008

Copyright (c) 2008 Jianwei Huang et al. This is an open access article distributed under the Creative Commons Attribution License, which permits unrestricted use, distribution, and reproduction in any medium, provided the original work is properly cited.

The advances of Internet and wireless access technologies have opened up new opportunities to serve high quality, ondemand multimedia applications. Applications like mobile TV, IPTV, on-demand streaming, and peer-to-peer video sharing have fundamentally changed the content distribution landscape, and have been accelerating a social and engineering revolution in media distribution and consumption. To achieve the ultimate goals of total freedom in selfexpression, seamless mobile access, and anytime anywhere media consumption, technology advances in various areas need to be reexamined and jointly utilized under a coherent optimization framework to reach an efficient end-toend media delivery solution. New models, metrics, and methodologies in source, and channel coding, distributed and collaborative communications are needed to intelligently adapt the multimedia content to suit user preferences, meet device and network constraints, and achieve better communication resource utilization. The source coding and adaptation decisions of media sources need to be reconciled with the limited network resources, end-user preferences, and resource allocation schemes at network nodes. Distributed optimization schemes like pricing and game theoretical approaches are needed to improve resource allocation and management efficiency. In this special issue on multimedia networking, we present several papers that address such issues.

The first paper of this special issue, "A collaborative wireless access to on-demand services" by Z. Naor, presents a collaborative access scheme that exploits the broadcast nature of the wireless communications in order to achieve better multicast content delivery. The proposed method is particularly suitable for sessions of long time durations, for applications where clients can subscribe to ahead of time, and for applications in which the clients receive the same information simultaneously.

The second paper of this special issue, "A stream tapping protocol involving clients in the distributions of videos on demand" by S. Kulkarni et al., presents a stream tapping protocol that involves clients in the video distribution process. Compared with the traditional taping protocol, the proposed one greatly reduces the workload of the video server by delegating part of the content distribution process to the clients who are watching the video.

The third paper of this special issue, "Automatic bandwidth adjustment for content distribution in MPLS networks" by D. Moltchanov, discusses a new algorithm for dynamic resource adaptation to temporarily changing traffic conditions in multiprotocol label switching (MPLS) networks. The major advantage of the proposed approach is that it is fully autonomous, takes into account statistical characteristics of traffic patterns, and is independent of the choice of the sampling interval of MPLS automatic bandwidth adjustment capability.

The fourth paper of this special issue, "Rate-distortion optimized frame dropping for multiuser streaming and conversational video" by $\mathrm{W}$. Tu et al., considers ratedistortion optimized strategies for dropping frames from multiple conversational and streaming videos sharing limited network node resources. Experimental results show that a significant improvement in end-to-end performance is achieved compared to priority-based random early dropping schemes. 
The fifth paper of this special issue, "A theoretical framework for quality-aware cross-layer optimized wireless multimedia communications" by $\mathrm{S}$. Ci et al., presents a theoretical framework for integrated cross-layer control and optimization in wireless multimedia communications. The framework includes two essential parts: a delay-distortiondriven optimization framework and a new approximate dynamic programming technique based on significance measure and sensitivity analysis for high-dimensional nonlinear cross-layer optimization.

The sixth paper of this special issue, "Optimal multilayer adaptation of SVC video over heterogeneous environments" by T. Thang et al., proposes an optimized framework of controlling the SNR scalability across multiple spatial layers in scalable video coding format. The proposed framework has the flexibility in allocating the resource (i.e., bitrate) among spatial layers, where the overall quality is defined as a function of all spatial layers' qualities and can be modified on the fly.

\section{ACKNOWLEDGMENTS}

We thank all the authors for their contributions to the special issues and wish the readers a pleasant reading. We would also like to thank the reviewers for taking time to complete the reviews promptly. 

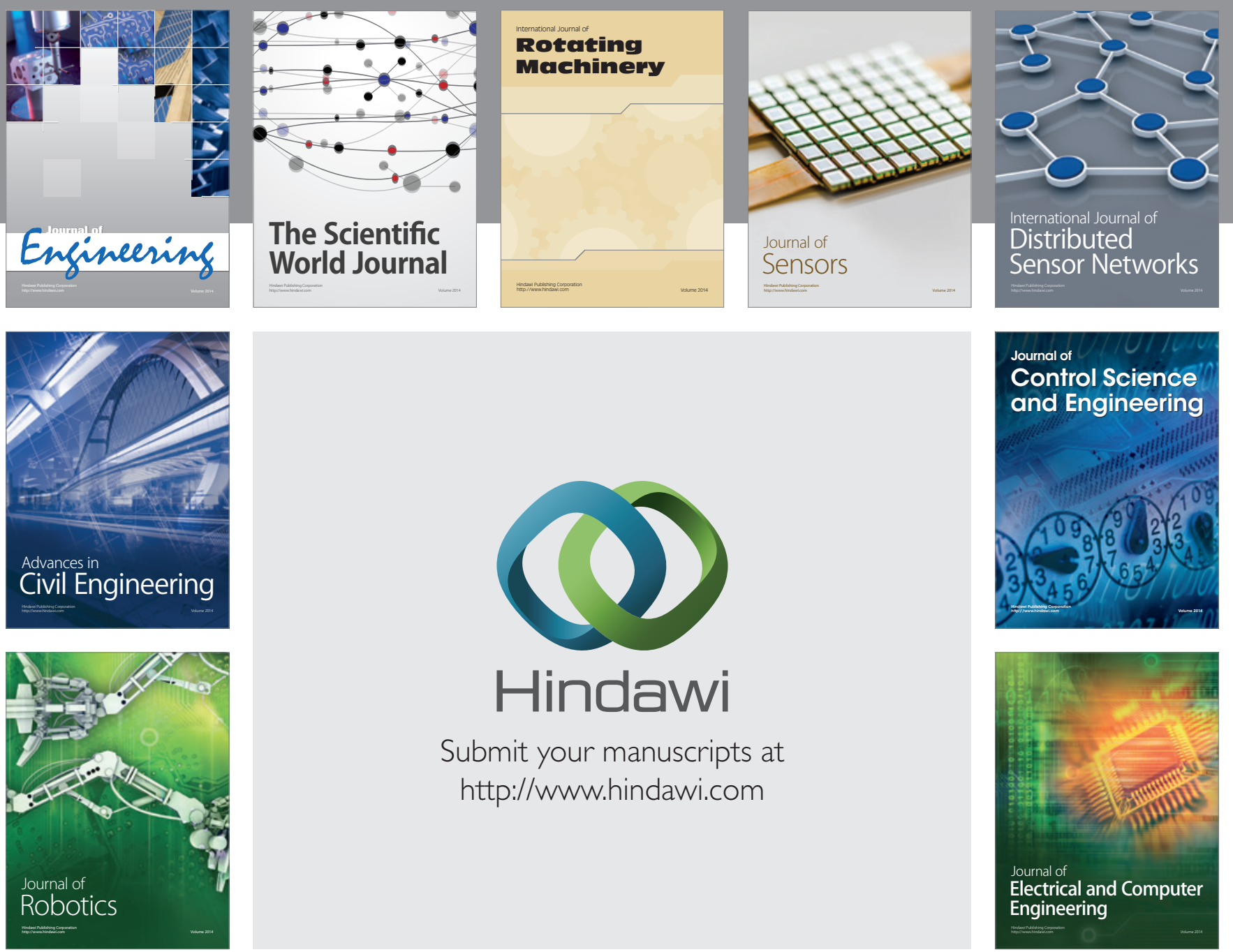

Submit your manuscripts at

http://www.hindawi.com
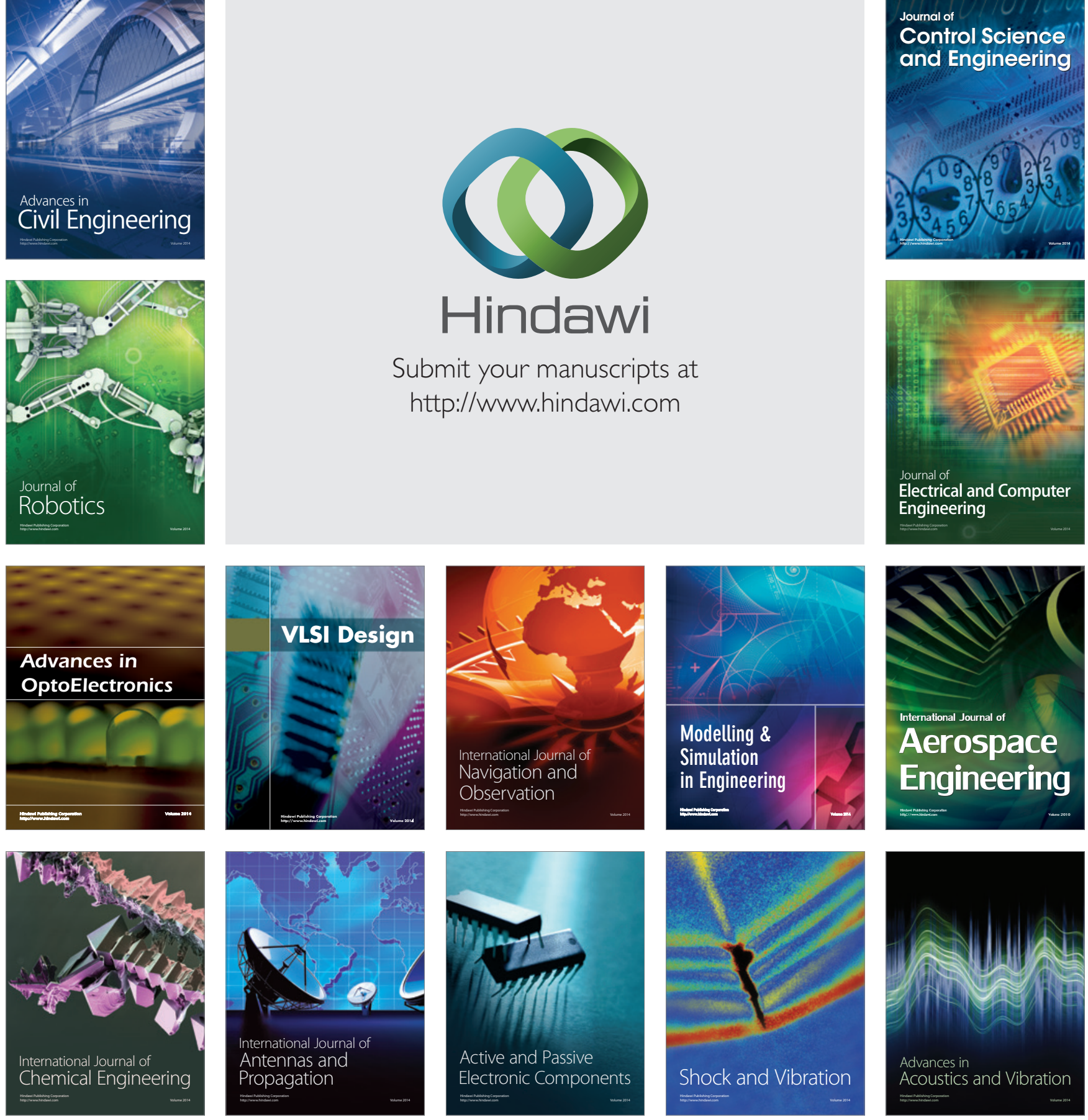